\title{
Making TTCN-3 testing a success
}

Key issues for an efficient tool environment

\author{
Wolfgang Sachse \\ Danet GmbH, Mobile Communications Technology \\ Gutenbergstrasse 10, D-64331 Weiterstadt, Germany \\ wolfgang.sachse@danet.de
}

\begin{abstract}
Now that the TTCN-3 standard has matured, first tools are appearing on the market. But the enhanced capabilities of TTCN-3 can only be fully exploited in a smart, flexible and open test environment.

This contribution from a test tool manufacturer's point of view highlights some of the key features and tool aspects that users of TTCN-3 should consider when setting up their test environment.
\end{abstract}

Key words: TTCN-3, tools, compiler, TRI, ASN.1

With TTCN-3, the 'Testing and Test Control Notation' of ETSI (European Telecommunication Standardisation Institute), a new global standard for a test specification language has turned up. TTCN-3 is not only 'just another revision' but a completely modernised edition of the wellproven test language which is now aiming at an additional deployment in the datacom domain. ETSI members have expressed their support by deciding early that TTCN-3 will be used for the development of test specifications for the voice-over-IP protocols SIP and OSP.

Its predecessor, TTCN-2++, is widely used in the telecommunications area for black-box system testing and conformance testing. Its particular merits are the integration in a framework of testing methodology and supporting standards, the independence from vendors and test systems and the availability of test suites, e.g. for DECT, UMTS and Bluetooth technologies.

TTCN-3 was meant to keep all of these advantages, and substantial effort was made to make the new version fit for additional testing applications by 
adding support for e.g. distributed systems, client server architectures (e.g. CORBA-based), and type/value systems (IDL and other programming languages). Also, the readability has been enhanced by creating a textual presentation format, called 'Core Notation', that looks like a 'normal' programming language. Other notations to specify tests comprise a tabular and graphical format. Now it is up to the test community to take advantage of the powerful new test capabilities that TTCN-3 is offering.

But all the benefits and the strength of such a powerful language can only be realised when it is supported by smart and efficient tools that allow users to concentrate on their basic tasks and to reach their goals in all phases of the testing process quickly.

The requirements for a TTCN tool environment are manifold as there are different types of users focusing on several different aspects. Therefore, beside the basic technical merits, the usability aspects and the openness and flexibility of a tool solution are very important for success and acceptance of the new test methodology. Based on Danet's rich experience over the past decade with its own TTCN test solution, called TTCN Toolbox, a closer look on some tool aspects and key requirements for a TTCN-3 test solution is taken in the following sections.

A general comment about suitable test solutions would be that not only the technical merits and features of the TTCN compiler are key issues, but also seamless tool integration and ease of use account for a good overall TTCN-3 testing solution.

\begin{tabular}{|c|c|c|c|c|}
\hline $\begin{array}{c}\text { Test } \\
\text { Development }\end{array}$ & $\begin{array}{c}\text { Test } \\
\text { Generation }\end{array}$ & $\begin{array}{c}\text { Test } \\
\text { Execution }\end{array}$ & $\begin{array}{l}\text { Result } \\
\text { Analysis }\end{array}$ & $\begin{array}{c}\text { Test } \\
\text { Reporting }\end{array}$ \\
\hline $\begin{array}{l}\text { - Test } \\
\text { conception } \\
\text { - Create Test } \\
\text { Environment } \\
\text { - Develop own } \\
\text { test suite } \\
\text { - Edit and } \\
\text { modify } \\
\text { existing test } \\
\text { cases } \\
\text { - Convert 'old' } \\
\text { TTCN-2 test } \\
\text { cases to } \\
\text { TTCN-3 }\end{array}$ & $\begin{array}{l}\text { - Semantic and } \\
\text { syntax checks } \\
\text { - Support for } \\
\text { TTCN-3 base } \\
\text { features } \\
\text { - Support for } \\
\text { test interface } \\
\text { libraries } \\
\text { - Generate and } \\
\text { compile } \\
\text { executable } \\
\text { code }\end{array}$ & $\begin{array}{l}\text { - Assign } \\
\text { parameter } \\
\text { values and } \\
\text { test ports } \\
\text { - Select test } \\
\text { cases for } \\
\text { execution } \\
\text { - Provide run- } \\
\text { time options } \\
\text { - Run and } \\
\text { supervise test } \\
\text { campaign } \\
\text { - Display results }\end{array}$ & $\begin{array}{l}\text { - Display trace } \\
\text { files } \\
\text { - Visualise trace } \\
\text { data } \\
\text { graphically } \\
\text { - Provide good } \\
\text { support for } \\
\text { fault-finding } \\
\text { - Support for } \\
\text { message } \\
\text { decoding } \\
\text { - Support for } \\
\text { debugging }\end{array}$ & $\begin{array}{l}\text { - Provide } \\
\text { statistic data } \\
\text { - Generate test } \\
\text { reports and } \\
\text { statements } \\
\text { - Generate } \\
\text { alarms } \\
\text { - Provide } \\
\text { access to } \\
\text { network } \\
\text { management } \\
\text { systems }\end{array}$ \\
\hline TTCN-3 & & & & 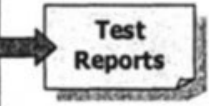 \\
\hline
\end{tabular}

Figure 1. Test phases overview. 
As depicted above, the testing process can be divided into five major phases that all require support by tools aiding the user and off-loading him from routine or unnecessary tasks.

The first major step is the Test Development phase where the test environment is created and the test cases are written. In particular, the question of how the test cases 'talk' to the implementation under test is of interest as here the testing infrastructure needs to be implemented. This communication might be implemented using simple mechanisms like a TCP connection or a serial link, in more complex cases an interface towards CORBA or proprietary protocol stacks might be required. Here it is important that the TTCN tool environment supports all those possibilities using an open mechanism that allows to make use of a well defined interface and providing sample implementations. ETSI has defined TRI, the 'TTCN-3 Runtime Interface' along with TTCN-3 to facilitate a standardised communication mechanism. Therefore it is a must for a TTCN test solution to provide a communications library based on TRI and to actively support users.

The second important point in the Test Development phase, of course, is the generation of a test suite and the test cases contained. Since many users still have a large stock of 'old' test cases that they want to continue to use and because TTCN-3 differs much from the TTCN-2 notation, it is crucial to provide a migration tool that automatically converts TTCN-2 test suites into TTCN-3. All further work can then be based on those migrated test cases, thus protecting the investment in previous test case development.

For modifying and writing own test cases, editing capabilities should be available. TTCN-3 now supports a textual presentation format that is easily readable for humans, looking mostly like C-code. This so-called 'Core Notation' is the common exchange format for TTCN-3. Therefore an integrated text editor (with syntax highlighting and further language support) is highly desirable. Beside the 'traditional' tabular presentation format which requires a specialised editor, TTCN-3 test specifications can also be 'designed' in a graphical presentation, called Test Sequence Charts, TSC. Whatever presentation format is used for the test case development, it is important that the use of editors is well integrated into the tool solution, even if third-party products are employed. A good integration provides for 'hyperlinking' from e.g. the TTCN compiler's error window or from the postexecution trace display back to the source code representation of the referenced object.

The second major step in the test process is the Test Generation phase where the TTCN-3 source code is translated into executable test programs. In this step, the power and features of the TTCN compiler define the quality of the test solution. The obvious issues, namely supported functionality and 
technologies are rather easy to decide: if a particular requirement cannot be met, the solution is not suitable. This already starts with the simple question of supported platforms: Does the tool solution run in the preferred (or intended) environment? Can test code be generated for UNIX, Windows, real-time environments or, using cross-compilers, for rather 'exotic' test environments? Other relevant aspects in this area are the support of TTCN-3 language elements and supporting technologies. For example, a comprehensive TTCN-3 solution in the telecom domain must support the data description language ASN.1 (X.680, '97) with the associated encoding rules (BER, PER, X.690). Particularly for a deployment in the datacom and CORBA world, IDL should be supported. Also ETSI's Test Synchronisation Protocol, TSP.1 would be desirable feature, particularly for testing in a distributed environment.

Apart from those rather easy-to-decide features, the implementation details of the TTCN-3 code generator significantly determine the quality and strength of the whole test solution. Flexibility and openness definitely enhance a test tool as these offer additional influence on the system's behaviour.

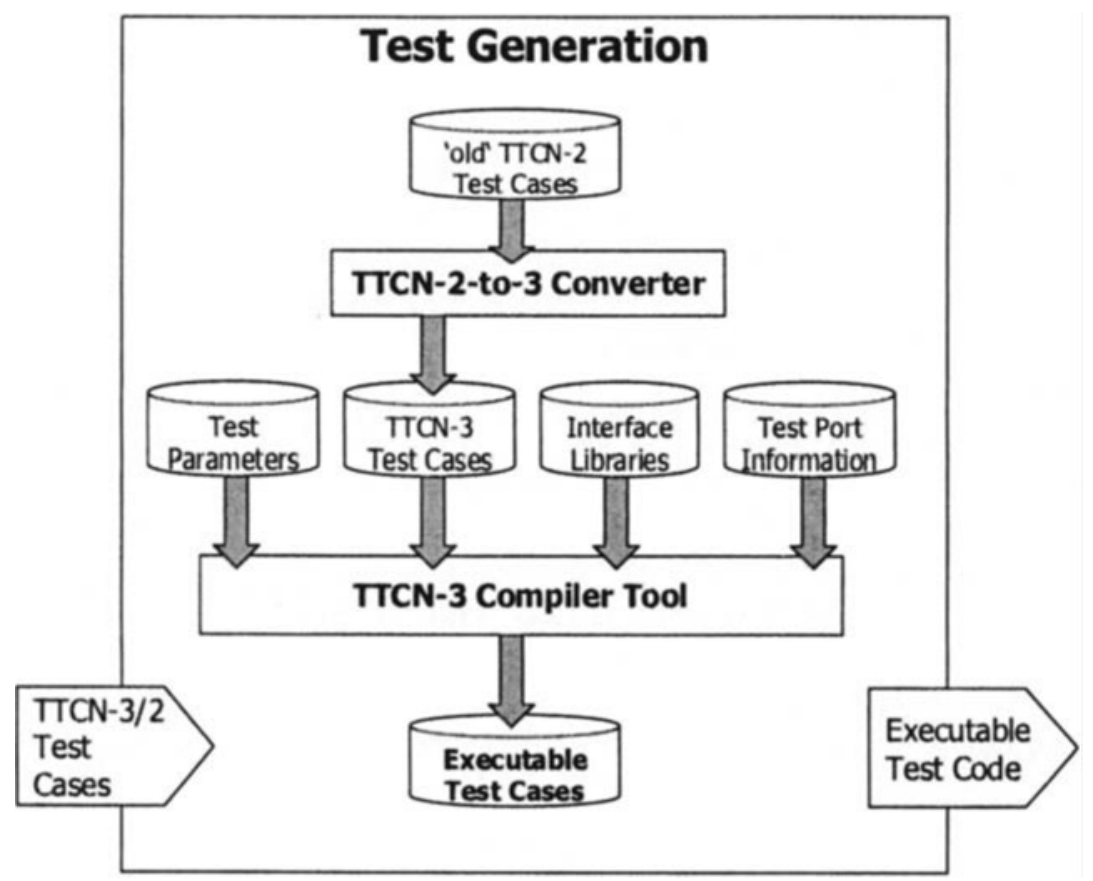

Figure 2. Test Generation phase.

In a first step of the Test Generation phase, test suites are parsed and analysed for syntactical and semantic correctness. A good compiler should 
check a test suite very rigidly because any faults or 'flaws' not discovered at this level may lead to very hard-to-find errors during the test execution phase. At worst, a test case may not perform its intended purpose at all!

Generally, not only for performance reasons, the translation from TTCN3 to executable code is performed by translating TTCN-3 into an intermediate 'standard' language. In Danet's TTCN Toolbox, for example, $\mathrm{C}++$ code is generated here. Using this two-step approach has several advantages: code can be compiled for virtually any target platform, existing libraries (e.g. for interface adaptations, available external functionality) can be linked to the test programs and standard compiler control and code optimisation tools can be used to fine-tune the generated code.

The speed of the test generation process and the size of the generated executables, however, should not be underestimated as these factors heavily influence the turn-around times perceived by the test case developer. Incremental compilation (only re-compiling recent changes in TTCN test modules), selective compiling (only compiling relevant test cases) and the possibility to use dynamically linked libraries improve the tool usability and user acceptance significantly.

Another advantage of the described two-step code generation process is the possibility to re-use the generated intermediate $\mathrm{C}++$ code for other purposes. For example, complete (!) functions for message de- and encoding are generated automatically based on the protocol knowledge the compiler got from the test specification. Also, adding additional protocol knowledge facilitating de-/encoding of under-defined message codings should be supported by a good tool solution. This would also allow to implement coders for proprietary encoding rules (such as CSN.1). Beside the automatic generation of de-/encoders, available external protocol-specific coders should be appliable.

Last but not least in the Test Generation phase, the administration of the generated code needs to be performed. Where shall the test programs be put, into a database, in a file system, in a repository? Since these requirements cannot be met at the same time in a standard product, the tool vendor should be able to offer customised solutions upon request.

The third major step in the test process is the Test Execution (or test campaign) phase where the user wishes to run the previously generated test programs, either to verify them (test case developer) or to perform the actual testing tasks (tester). But before running a test case, the user needs to assign parameters and test ports, based on the current test environment's settings. Also, some options for the execution of the test campaign may be required to set. These tasks should be supported by dedicated tools in the TTCN-3 test solution. 
Since at compile time only the test parameter names and types are known, but not their values, this assignment needs to be made just before run-time. This should be performed using a test suite parameter editor provided by the test campaign manager. Also the assignment of logical test ports (from the test suite's point of view) to their physical implementations needs to be supported by a tool.

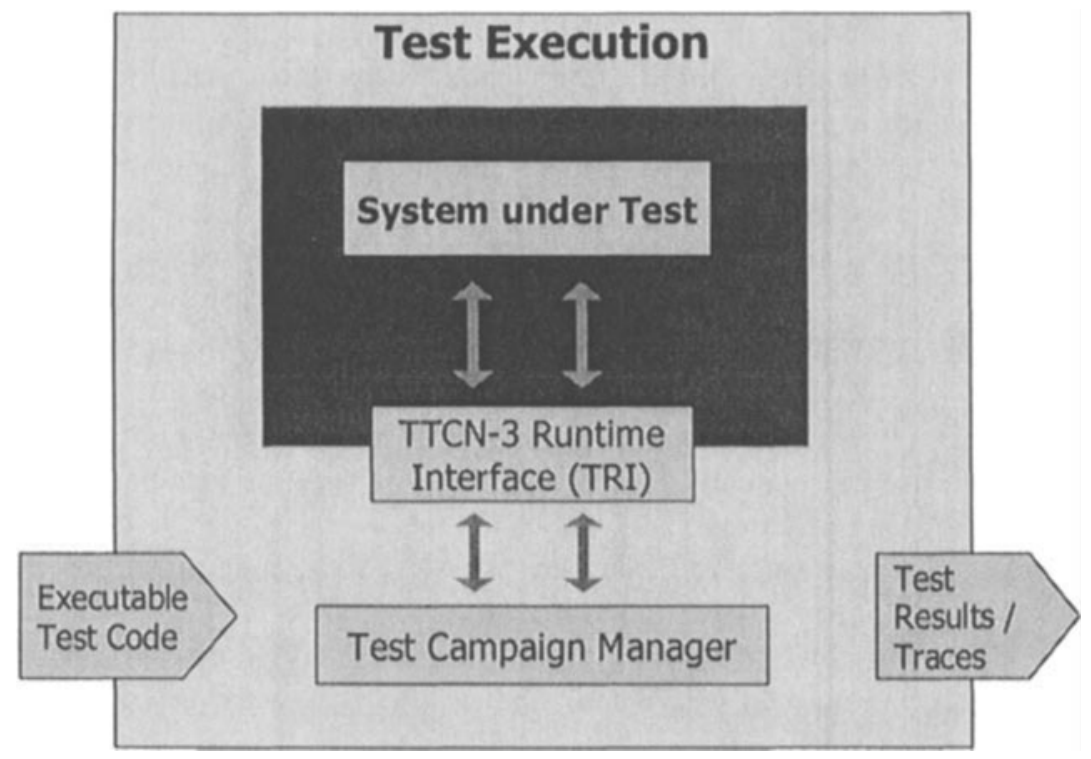

Figure 3. Test Execution phase.

For the definition of a test campaign and the selection of contained test cases, a good representation of the test suite's structure and possibilities to interactively select test cases would be very helpful. Alternatively, making use of the new 'control' structure of TTCN-3 that allows to influence the test execution in a script-like way is a desirable feature of a flexible test solution. Some features supporting e.g. 'timed' execution of a test campaign and the display of verdicts and the current status of the execution are improving the use of the test campaign management.

The fourth major step in the test process is the Result Analysis phase. This phase becomes particularly important for test cases that did not 'pass' as expected. Then a tool-based analysis of the results obtained from the test execution should support the user to pinpoint the problem.

A well-structured trace display function on TTCN level gives a good overview and provides information about the overall behaviour of the test case. It shows all relevant dynamic actions of all test components of a test case and provides the user with additional, useful information by fully decoding messages and showing variable assignments. Very helpful is the 
ability to 'hyperlink' from a line in the trace window directly to the associated TTCN source code statement, highlighted by the TTCN editor. This provides a means for quick turn-around times and efficient fault-finding facilities. Complementary to the textual traces is a graphical representation of the trace information in sequence diagrams, e.g. as Message Sequence Charts, MSC. This way of displaying the trace information is more intuitive for human users and also offers a better overview of the events during a test case's run.

Apart from comprehensive tracing facilities, a good result analysis tool should offer support to locate a problem and assist a user in finding the cause for a 'deviation' from the expected result. Hence, after guiding the user to such a location of interest, additional information displaying the actual and the expected result will reveal what exactly caused a particular verdict.

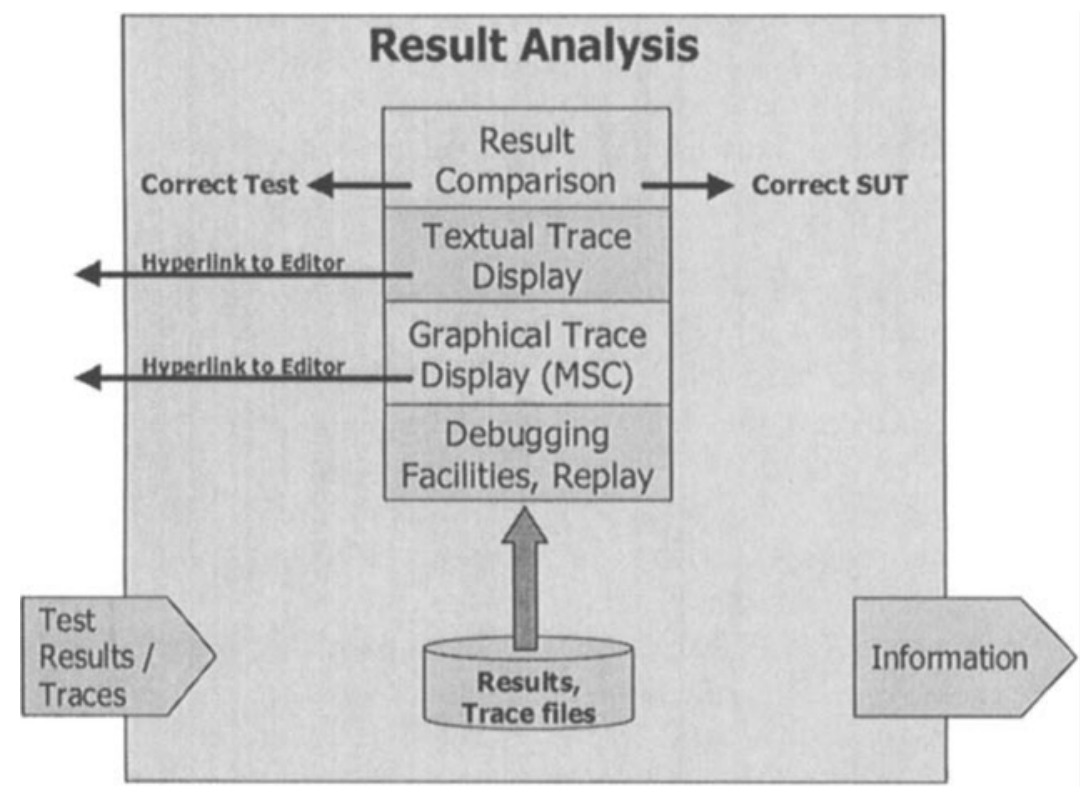

Figure 4. Result Analysis phase.

Debugging facilities may prove useful to identify hard-to-find problems with a test suite, especially when applied by advanced users. To get around the real-time problems of low-level debugging, 'recording' a test case's dynamic behaviour and subsequently re-playing this trace data back to the test program is a smart solution. This allows to simulate the behaviour while offering extensive off-line debugging facilities and avoiding timer problems.

The last major step in the test process is the Test Reporting phase which mainly comprises functions for automated post-processing and evaluation of test execution results. Unlike in the previous phases where the test tool's 
functions are mostly supporting user interactions, the reporting phase mostly off-loads the user from tiresome routine tasks. Particularly for conformance testing and similar test applications, the generation of standard reports and the documentation of test results is a must. In test environments for monitoring purposes, it is vital to automatically generate alarms based on particular test results. Hence, interfaces to network management systems (in the case of telecom applications) or other alarming services are additional benefits.

Regarding the documentation support, a good and flexible tool should provide access to the information gathered during the test campaign in an open format. This information may comprise the test case execution information, obtained verdicts, information about the test configuration, the traces files, the test suite parameters and other collected or logged data. By importing, post-processing and transforming this information into custom reports, most user's demands for result documentation and management information systems should easily be met.

Going a bit further than just providing the raw data that can be processed into reports or templates in a second step, the test tool vendor may offer to generate the required reports directly in a customer-specific format.

So, after all, what makes TTCN-3 testing a success? As one can see from the above considerations, a good TTCN-3 test solution is much more than just a few 'ticks' in a buzz word feature list. It is the tool vendor's experience and the user feedback, together with a flexible and open architecture that define the quality of the overall test solution and hence, the user's testing success.

Based on Danet's experience with its TTCN Toolbox product, also customer support and the 'surrounding' services offered to the user contribute to the success of a TTCN-3 test environment, apart from the pure technical merits of the test solution, of course. 